\title{
PROTECCIÓN DEL PATRIMONIO CULTURAL Y ARQUEOLÓGICO DEL DISTRITO DE CURUBANDÉ, CANTÓN DE LIBERIA, MEDIANTE EL PLAN DE DESARROLLO TURÍSTICO SOSTENIBLE
}

\author{
Félix Zumbado Morales \\ Programa de Investigación en Desarrollo Urbano Sostenible (ProDUS) \\ Universidad de Costa Rica
}

Recibido: $25-10-2016$

Aprobado: 25-03-2016

Ing. Agr. Félix Zumbado Morales. MSc. Colaborador en el Programa de Investigación en Desarrollo Urbano Sostenible (ProDUS) de la Universidad de Costa Rica, investigador en temas de ordenamiento territorial, sector agropecuario, desarrollo rural, turismo sostenible, ecoturismo. Coordinador del proyecto de elaboración del Plan de Desarrollo Turístico Sostenible del distrito de Curubandé y del cantón de Guatuso.

felzum@gmail.com

\section{RESUMEN}

Durante el periodo 2014-2015 el Programa de investigación en Desarrollo urbano Sostenible de la Universidad de Costa Rica realizó el proyecto "Plan de Desarrollo Turístico del cantón de Guatuso" y "Plan de Desarrollo del distrito de Curubandé, cantón de Liberia". Proyecto financiado por el Banco Interamericano de Desarrollo BID y gestionado por el Sistema Nacional de Áreas de Conservación SINAC, la Municipalidad de Guatuso y la Municipalidad de Liberia. El objetivo del proyecto era generar una propuesta que permitiera fomentar el desarrollo de turismo rural. Como resultado del diagnóstico se encontró destrucción de algunos de los Petrograbados que se ubican en las márgenes del río Colorado. Por esta razón el equipo de ProDUS presentó la correspondiente denuncia ante el Museo Nacional y las autoridades de la Municipalidad de Liberia. El Museo Nacional realizó una gira de reconocimiento y generó un documento con directrices para la preservación del patrimonio. Posteriormente se presentó el documento a las autoridades del Municipio, quienes deberán generar las acciones necesarias para evitar la destrucción del recurso. Este documento se centra en las lecciones aprendidas que serán de utilidad para otros proyectos donde se incluya el componente del patrimonio cultural como un elemento central de un Plan de Desarrollo Turístico Sostenible.

Palabras Clave: Curubandé, Petrograbados, Conservación y Patrimonio 


\section{ABSTRACT}

The project was developed for the Sustainable Urban Development Research Program at the University of Costa Rica ProDUS- UCR during the period 2014-2015. The aim of the project was to promote the development of rural tourism in Curubandé. As result of the process of diagnosis the equipment of investigators found the destruction of some of the petroglyph located in the Colorado river. For this reason, the equipment of ProDUS presented the denunciation to the authorities of the Costa Rica National Museum and to the personal of the Municipality of Liberia. The authorities of the National Museum realized the inspection and generated a document with directives for the preservation of the heritage. The document was sent to the municipal authorities, who will have to generate the necessary actions to avoid the destruction of the resource. This paper sets out the most common themes that were identified as lessons to be learned.

Keywords: Curubandé, petroglyph, heritage conservation

\section{INTRODUCCIÓN}

El proyecto Plan De desarrollo turístico del distrito de Curubandé y del cantón de Guatuso es resultado del esfuerzo del equipo de trabajo interdisciplinario de ProDUS-UCR. El componente del estudio del patrimonio arqueológico del proyecto fue desarrollado por la Licenciada en Antropología Paola Salazar Arce, quien era funcionaria de ProDUS en el momento de realizar la investigación.

El trabajo incluyó la elaboración de un diagnóstico general del distrito. Como parte de los resultados se encontró destrucción y alteración de los Petro grabados ubicados en las márgenes del río Colorado. A continuación se presentan datos relevantes sobre la metodología y los resultados relacionados con el tema de patrimonio cultural y arqueológico.

\section{Metodología de trabajo del proyecto:}

El proceso de elaboración del proyecto constó de las etapas de diagnóstico, elaboración de políticas públicas y generación del Plan.

La fase de diagnóstico incluyó la realización de investigaciones en las temáticas de: uso del suelo en la zona, zonas de vida, áreas silvestres protegidas y planes de manejo, conflictos ambientales, topografía, capacidad de uso del suelo, amenazas naturales, actividades productivas, indicadores demográficos, indicadores de vivienda y pobreza, indicadores de empleo, indicadores de seguridad ciudadana, descripción de los poblados, sistema educativo de la zona, educación y capacitación 
relacionada con turismo en la zona, percepción del turismo en las comunidades del distrito, patrimonio arqueológico y cultural, sistema turístico, red vial y transporte, energía, agua potable, manejo de aguas pluviales y de aguas residuales, salud, desechos sólidos, telecomunicaciones, actores clave, instrumentos de planificación en la zona.

Posterior a la etapa del diagnóstico se realizó la etapa de Políticas Públicas, que consistió en el mapeo de instituciones públicas que trabajan en el área de estudio, posteriormente se realizó el análisis de las responsabilidades según la ley que tienen esas entidades y por medio de entrevistas y grupos focales se establecieron los proyectos actuales y futuros de estas organizaciones. La información obtenida permitió establecer los proyectos en los que participarán cada una de las organizaciones con responsabilidades dentro del Plan de Desarrollo turístico, estableciendo para cada proyecto un plazo, indicadores de comprobación y responsables de la ejecución y seguimiento.

La etapa final del trabajo consistió en la generación del documento del Plan, donde se establecieron los proyectos que conformaron el Plan de Desarrollo turístico, donde se estableció que era necesario la realización del Plan Regulador del cantón de Libería, construir un centro comunal administrado por la Asociación de Desarrollo de Curuabandé donde se ubicará el restaurante de comidas tradicionales; un museo comunitario; centro artesanal y una plaza de actividades culturales. Otros de los proyectos son miradores para los Petrograbados ubicados en el río Colorado, puente colgante peatonal para comunicar con un mirador ubicado en la zona del Escobio, plataforma de observación en las afueras del proyecto Pailas II y ciclovias. 


\section{Componente patrimonial dentro del proyecto Plan de Desarrollo Turístico del distrito de Curubandé}

En este apartado de la investigación incluyó el análisis del patrimonio arqueológico y cultural intangible para el distrito de Curubandé de Liberia, a partir de información bibliográfica recopilada respecto a investigaciones arqueológicas realizadas en la zona. Se logra describir y caracterizar la riqueza arqueológica del distrito mediante los registros del Museo Nacional de Costa Rica (MNCR), así como su importancia dentro de la zona de estudio.

Para el caso del patrimonio cultural intangible se elaboró un inventario del acervo cultural local por medio de entrevistas a profundidad dirigidas a pobladores de Curubandé. Lo anterior, permitió una triangulación de fuentes de datos primarias y secundarias, con el fin de comprender la realidad sociocultural y las posibles transformaciones de las expresiones inmateriales en la zona, como lo son: las prácticas productivas, la comida tradicional basada en maíz, la práctica de medicina tradicional, la elaboración de artesanías con productos locales y la música popular. Además de la presencia del patrimonio arqueológico y la evidencia de resabios históricos como los petrograbados. (ProDUS, 2015)

Complementariamente, se desarrolló un proceso consultivo a pobladores de las comunidades aledañas a Curubandé, por medio de una encuesta se consultó a 32 personas que viven en el distrito de Curubandé. La división de personas encuestadas por poblados es la siguiente: 20 personas de la comunidad de Curubandé; 7 personas del poblado El Gallo; 4 vecinos de Guadalupe y 1 persona de la comunidad de San Rafael (La Choricera). En el tema de cultura y gastronomía se preguntó por recetas o platillos tradicionales de la zona y patrimonio oral, esto con el fin de incorporar este elemento dentro de las propuestas del Plan de Desarrollo Turístico.

\section{Tradición oral de la zona}

El rescate del patrimonio intangible fue uno de los ejes transversales del trabajo, buscando incorporar en los proyectos componentes relacionados con este eje. A continuación se presentan los resultados de las preguntas relacionadas con el tema cultural incluidas en la encuesta realizada a los pobladores de las comunidades de Curubandé. 


\begin{tabular}{|c|c|c|}
\hline Leyenda & $\begin{array}{c}\text { \# de personas que mencionaron } \\
\text { la leyenda }\end{array}$ & $\begin{array}{c}\text { \% del total de } \\
\text { entrevistados }\end{array}$ \\
\hline Curubanda & 12 & $38 \%$ \\
\hline La Mona & 5 & $16 \%$ \\
\hline La llorona & 5 & $16 \%$ \\
\hline La Carreta sin Bueyes & 3 & $9 \%$ \\
\hline El Gallo & 1 & $3 \%$ \\
\hline La Cegua & 1 & $3 \%$ \\
\hline Tulevieja & 1 & $3 \%$ \\
\hline Casona Santa María & 1 & $3 \%$ \\
\hline
\end{tabular}

Tabla 1.Leyendas o cuentos mencionadas por los encuestados.

Fuente: ProDUS-UCR (2014)

Las leyendas o cuentos forman parte del patrimonio intangible de los pueblos. Este legado de tradición generalmente oral conforma una riqueza que se va perdiendo conforme los pueblos pierden su identidad y se presentan fenómenos de migración y transculturización.

La tabla 1 muestra las leyendas que conocen los entrevistados. Estas leyendas son una oportunidad para integrarlas en el paquete turístico de la zona. El nombre de la comunidad Curubandé viene de una leyenda de origen indígena y no hay ningún símbolo que indique al viajero este hecho. En países como México, Guatemala o Perú se ha logrado incorporar en el paquete turístico este patrimonio intangible. Una muestra de cómo se ha desarrollado este proceso es el mercadeo de los pueblos mágicos dentro de la oferta de turismo mexicana. Esto es de importancia para relacionarse con las leyendas que forman parte de la tradición oral del lugar. 


\begin{tabular}{|c|c|}
\hline Platillos tradicionales & $\begin{array}{c}\text { \# de personas que lo } \\
\text { mencionaron }\end{array}$ \\
\hline Arroz de maíz & 11 \\
\hline Gallo pinto & 7 \\
\hline Tortilla dulce & 5 \\
\hline Cuajada & 5 \\
\hline Tortillas & 3 \\
\hline Tamales & 3 \\
\hline Pozol & 3 \\
\hline Cajetas & 2 \\
\hline Rosquillas & 2 \\
\hline Gallos de gallina & 2 \\
\hline Atol pujagua & 1 \\
\hline Vigorones & 1 \\
\hline Plátanos maduros & 1 \\
\hline Chicheme & 1 \\
\hline Coyoles & 1 \\
\hline Arroz con pollo & 1 \\
\hline Pinol & 1 \\
\hline
\end{tabular}

Tabla 2.

Resumen de las recetas tradicionales mencionadas por los encuestados.

La gastronomía local es un elemento de importancia en el desarrollo de los paquetes de turismo sostenible. Se les consultó a los entrevistados su opinión sobre platos tradicionales de la zona. La tabla 2 muestra los platillos que se mencionaron en las respuestas.

La primera conclusión a la que se llega con la información de la tabla 2 es que la mayor parte de los platillos con mayor potencial para incluirse en un restaurante de comida tradicional no están a la venta en la zona de Curubandé. El gallo pinto, arroz con pollo y cajetas son los únicos alimentos que sería posible adquirir en la zona. Platos como el arroz con maíz, la tortilla dulce, la cuajada, el pozol, gallos de gallina, atol pujagua, vigorones, chichime o coyoles no están en la oferta gastronómica disponible para el visitante. Esto representa un gran potencial para instalar un restaurante de comida tradicional. 
Los motivos por los que no se ofrecen estos platillos en los menús de los restaurantes son diversos y no se puede dar una sola causa. Dentro de algunas de las variables que influyen en que no estén disponibles estos platos en los servicios de ventas de alimentos son:

- Falta de interés en integrar estos platos en la oferta de los locales

- Desconocimiento de las recetas y su preparación debido a que son solo conocidas por algunas personas de la comunidad

- Temor a que no sea rentable su incorporación en los menús

- Temor a que los turistas no gusten de los platillos

- No se ha evaluado el potencial que tienen estos platillos como complemento a la oferta gastronómica actual

- Desconocimiento de su potencial como parte del atractivo turístico de la zona

\section{Generalidades sobre el componente arqueológico}

El hallazgo arqueológico es un elemento cultural de gran valor que debe ser manejado y proyectado adecuadamente. El beneficio que obtiene el distrito de Curubandé con esta herencia se traduce en el enriquecimiento de su identidad cultural; la cual se comparte con otros grupos humanos cotidianamente.

Al respecto, se debe tomar en cuenta que la arqueología es una ciencia interesada en estudiar el pasado de los grupos humanos; desde las actividades humanas, hasta su conducta. Para obtener este conocimiento analiza el testimonio material que dejaron los distintos grupos culturales, que se asentaron en determinados territorios. Dicho conocimiento se construye a partir de cualquier vestigio, o expresión material reflejo del arte, la industria, la técnica y la forma de vida de los habitantes prehispánicos. (ProDUS, 2015)

Al respecto, la Ley sobre Patrimonio Nacional Arqueológico, (Ley N 6703) en su artículo $1^{\circ}$ considera patrimonio arqueológico nacional a los bienes:

"...muebles o inmuebles, producto de las culturas indígenas anteriores o contemporáneas al establecimiento de la cultura hispánica en el territorio nacional, así como los restos humanos, flora y fauna, relacionados con estas culturas". (Asamblea Legislativa, 1982). 
Por lo tanto, la salvaguarda de la herencia cultural de los antepasados es de vital importancia, no solo para comprender mejor la historia y reconstrucción del pasado, sino también para reforzar valores de la idiosincrasia costarricense. De acuerdo con esto, la identidad de las comunidades está estrechamente ligada a los elementos arqueológicos presentes en su territorio y su historia. (ProDUS, 2015).

\section{Sitios arqueológicos de Curubandé}

En el distrito de Curubandé el Museo Nacional reporta 27 sitios arqueológicos, los cuales presentan evidencia material, a partir de la cual se han determinado las cronologías de los sitios y su estado de conservación.

La información de los sitios se resume en la tabla 1.

En el lugar sobresalen los áreas de carácter petroglifo, habitacional y funerario; siendo este último el que más común. En el caso del sitio Zapote este es de tipo funerario con terrazas de bajo relieve, surcado por dos micro cuencas. Aquí también se han encontrado petro grabados elaborados en andesita.

Otro de los sitios arqueológicos de Curubandé en donde, igualmente, se han encontrado petro grabados es Hombre Mono ubicado cerca de una de las fumarolas del Parque Nacional Rincón de la Vieja, lo que hace suponer que el grupo humano que habitaba estas tierras mantenía ciertas prácticas y creencias que denotaban temor y respeto por el Volcán; además de que puede asociarse a la construcción de cementerios y sitios habitacionales. (ProDUS, 2015) 


\begin{tabular}{|c|c|c|c|c|c|}
\hline \multicolumn{3}{|c|}{ Información general } & \multicolumn{3}{|c|}{ Temporalidad } \\
\hline $\begin{array}{c}\text { Nombre del } \\
\text { Sitio }\end{array}$ & $\begin{array}{c}\text { Área } \\
\text { Estimada } \\
\left(\mathrm{m}^{2}\right)\end{array}$ & $\begin{array}{c}\text { Estado de } \\
\text { conservación }\end{array}$ & $\begin{array}{ll}0 & \\
\pi & \\
0 & \\
0 & \\
0 & \\
0 & 0 \\
0 & 0 \\
0 & \\
0 & \\
0 & \end{array}$ & 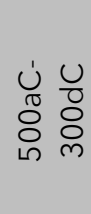 & 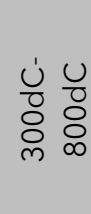 \\
\hline Bernabe & -- & -- & & & $x$ \\
\hline Camacho & -- & -- & $x$ & & \\
\hline Caucel & -- & -- & & & $x$ \\
\hline Cedral & -- & -- & & & $x$ \\
\hline Colorado & 3375 & Perturbación múltiple & & & $x$ \\
\hline Curubandé & -- & Perturbación múltiple & & & $x$ \\
\hline El Blanco & -- & Perturbación múltiple & $x$ & & $x$ \\
\hline Flechas & -- & Perturbación múltiple & $x$ & & $x$ \\
\hline Guácimo & -- & Perturbación múltiple & & & $x$ \\
\hline Higuerón & -- & -- & & & \\
\hline $\begin{array}{l}\text { Hombre } \\
\text { Mono }\end{array}$ & -- & -- & $x$ & & \\
\hline Justino & -- & -- & & & $x$ \\
\hline La Casona & -- & -- & & & $x$ \\
\hline La Española & -- & -- & $x$ & $x$ & \\
\hline Las Pilas & -- & Huaqueado & & & $x$ \\
\hline $\begin{array}{c}\text { Molino de } \\
\text { Agua }\end{array}$ & -- & -- & & & $x$ \\
\hline Ollas & -- & -- & & & $x$ \\
\hline Para & 194534 & Perturbación múltiple & & & $x$ \\
\hline Pachote & -- & Perturbación múltiple & & & $x$ \\
\hline $\begin{array}{c}\text { Quebrada } \\
\text { Victoria }\end{array}$ & 13609 & Perturbación múltiple & & & $x$ \\
\hline $\begin{array}{c}\text { Rincón de la } \\
\text { Vieja }\end{array}$ & 4187 & Perturbación múltiple & & & $x$ \\
\hline Rivas & -- & -- & & & $x$ \\
\hline Sendero & 5123 & Perturbación múltiple & & & $x$ \\
\hline Tempisque & -- & Perturbación múltiple & & & $x$ \\
\hline Vista del Mar & -- & -- & & & $x$ \\
\hline Zapote & -- & Pastizal & $x$ & & $x$ \\
\hline
\end{tabular}

Tabla .3 Sitios arqueológicos registrados en el distrito Curubandé Fuente: Departamento de Antropología e Historia, Museo Nacional de Costa Rica, 2014. Citado por ProDUS-2015. 


\section{Proceso de denuncia y acciones para protección del sitio:}

Durante el año 2015, el equipo de ProDUS-UCR realizó varias giras a la zona del río Colorado, el fin de las mismas fue analizar el estado de los Petrograbados del sitio y su potencial para incluirse como un elemento diferenciador dentro del Plan de Desarrollo Turístico del distrito. Los resultados del trabajo de campo muestran que se ha dado alteración de los Petrograbados, muchos han sido rayados con clavos o tornillos, otros se han alterado al escribir sobre ellos los nombres de visitantes o simplemente trazando líneas sobre los mismos. Ante esta situación se realizó la denuncia respectiva.

En el mes de mayo del año 2015 el equipo de ProDUS-UCR denunció ante las autoridades del Museo Nacional de Costa Rica la situación de alternación de Petrograbados encontrada. Como resultado del proceso, el personal del Museo Nacional realizó una inspección en el sitio y confirmó la alteración de la zona por la realización de grabados hechizos con piedras, cuchillos, clavos, lo que lleva según los expertos en arqueología del Museo Nacional de Costa Rica produce un falso histórico y algunos petro grabados han sido re trabajados, distorsionando su diseño original

Dentro de las recomendaciones que da la Licda. Leidy Bonilla Vargas, arqueóloga del Departamento de Protección Cultural del Museo Nacional quien se encargó de atender la denuncia se tienen:

- Se recomienda que la Municipalidad de Liberia y la comunidad de Curubandé se comprometan no sólo a proteger, sino más bien, a darle seguimiento a este tipo de iniciativas de gestión integral dentro de un programa anual que cuente con recurso humano y económico, y que no desaparezca en el corto plazo o cuando se dé un cambio de gobernantes. El Museo Nacional puede asesor, brindar información, hacer inspecciones, y actuar en la conservación preventiva o correctiva si fuera el caso.

- Es necesario que se tomen acciones urgentes para asegurar la protección del recurso, como parte del proceso, el equipo de ProDUS-UCR envió en el mes de noviembre del 2015 una nota informando del tema al alcalde de la Municipalidad de Liberia y al presidente del Concejo Municipal. 
- Otra de las recomendaciones es la colocación de señalización tanto desde la carretera como dentro del lugar. Que indique: el nombre del río, la comunidad cercana, el respeto al cuido del mismo, al ser un sitio arqueológico el que no se debe de intervenir ningún elemento que lo distorsione, y sería conveniente contar con vallas de seguridad en algunas áreas peligrosas.

- Esperar los resultados del proyecto de conservación de las esferas de piedra del sur de Costa Rica e incorporar las medidas encontradas en los programas de conservación de los petrograbados.

- Otro aspecto que se pudo observar es la gran cantidad de basura que los turistas dejan en el lugar, desde botellas, bolsas de golosinas y otros, hasta tarros y residuos varios, que malogran la impresionante vista del lugar. En este sentido sería bueno igual por medio de avisos escritos motivar a los visitantes a recoger los deshechos y no dejarlos en el lugar.

- Se recomienda que en la zona se coloque rotulación que incluya información geológica y de especies florales y de fauna para que el recorrido que rea realicé en la zona de los Petrograbados se complemente con estos datos.

Los Petrograbados de la zona deben ser protegidos, es necesaria la rápida intervención de las autoridades municipales para evitar el deterioro del sitio y para lograr un adecuado manejo de la visitación que se da en el lugar.

\section{Proyectos del Plan que incluyen el componente del patrimonio cultural:}

Por medio de los siguientes proyectos se logró incorporar el componente del patrimonio cultural:

- Diseño de un logo que identificará la comunidad y será utilizado en todos los productos elaborados por los habitantes del sitio, el diseño se desarrolló tomando como base el entorno natural y el patrimonio cultural 
utilizando como referente los Petrograbados y sus diseños y la leyenda de Curubanda.

- Incorporación del patrimonio gastronómico local para establecer un restaurante de administración comunitaria y que ofrezca a los visitantes platillos típicos de la zona y que fueron elaborados con ingredientes de la zona.

- Fomento de la creación de grupos de rescate de la cultura local, teniendo la posibilidad de hacer presentaciones y exposiciones en el centro comunitario propuesto en el Plan.

- Creación de un museo comunitario donde se rescate la cultura local, lo anterior dentro del marco del proyecto de Museos Comunitarios del Museo Nacional de Costa Rica. El museo será elaborado por la comunidad con ayuda del personal del Museo Nacional de Costa Rica.

- Construcción de un mirador en el río Colorado para poder observar los petrogabados de la zona, creando un espacio donde se pueda colocar información sobre la historia y contexto de estas expresiones culturales.

- Incorporación de los elementos culturales representativos de la comunidad en la rotulación y diseño de la infraestructura de la ciclo vía propuesta.

- Se logró el compromiso por parte de las autoridades de la Municipalidad de Liberia para contratar una persona que se encargue de la gestión del Plan y todo lo relacionado con la promoción cultural y del turismo en ese municipio. 
Al tercer trimestre del 2016, el personal del SINAC y la Asociación de Desarrollo de Curubandé están en la búsqueda del financiamiento para iniciar las obras del Plan, se espera que para el año 2017 se logre dar el primer paso para construir las primeras infraestructuras propuestas.

\section{COMENTARIOS FINALES}

Por medio del Plan de Desarrollo Turístico del distrito de Curubandé, cantón de Liberia se desarrolló una iniciativa que rescata elementos del patrimonio tangible e intangible de la zona y los incorpora dentro de las propuestas para incentivar el desarrollo rural sostenible del territorio.

Este tipo de proyectos permite a las comunidades rurales generar iniciativas que propicien el empleo local de la mano del rescate cultural. Dentro de las limitaciones de mayor peso para lograr que estos proyectos se realicen se encuentra el financiamiento de las iniciativas, a pesar del soporte técnico y de calidad de investigación que brinda un proyecto desarrollado desde la Universidad de Costa Rica se ha dificultado el proceso de conseguir los fondos para iniciar la construcción de las obras.

Es necesario que la sociedad costarricense genere mayores oportunidades para que las propuestas de desarrollo y rescate cultural de base comunal puedan obtener financiamiento, mediante la experiencia desarrollada con el Plan de Desarrollo Turístico del distrito de Curubandé se demostró la dificultad que se tiene para encontrar financiamiento para realizar los proyectos clave necesarios para fomentar el desarrollo local.

\section{BIBLIOGRAFIA}

Aranzazu, R. (2003). Formación para líderes de turismo: herramientas para la gestión sostenible de turismo. Colombia, $14 \mathrm{p}$

Bertonatti, C. (2007), "El guía de turismo ¿Puede ser intérprete del patrimonio?", en Fernández Balboa, C. (Comp.) La interpretación del patrimonio en la Argentina. Estrategias para conservar y comunicar nuestros bienes naturales y culturales. Buenos Aires; Administración Parques Nacionales, Asociación para la Interpretación del Patrimonio, pp. 111-122

CICATUR; 1979. Metodología de inventarios turísticos. México. 198 p. 
Comisión Europea de Turismo y Organización Mundial del Turismo (2013), Manual de desarrollo de productosturísticos, OMT, Madrid. 159 p.

Instituto Nacional de Turismo INA. 2002. Módulo de formación. Generalidades del Turismo. San José, Costa Rica. 58 p.

Jafari, J. (2000). Encyclopedia of Tourism. Great Britain. 682 p.

Jiménez, A. (2003). "Una aproximación sistémica al turismo: implicaciones para la multi y la transdisciplinariedad". Universidad de Caribe. México, 17 p.

Mendoza, M.; Umbral, M.; Nadxielii, M.; Moreno, A. (2011). La interpretación del patrimonio, una herramienta para el profesional del turismo. El Periplo Sustentable, núm. 20, enero-junio, 2011, , Universidad Autónoma del Estado de México, México. pp. 9-30

MINCETUR (2005). Conceptos Fundamentales del Turismo. Perú. 445 p

Leiper, Neil. (1995). Tourism Management. Victoria. TAFE Publications. 368p

Organización Mundial del Turismo OMT. (1993) Desarrollo Turístico Sostenible, Guía para planificadores locales. Madrid, España. 235 p

Olivera, A. (2011). "Patrimonio Inmaterial, recurso turístico y Espíritu de los Territorios". Cuadernos de Turismo, n²7, (2011); pp. 663-677 Universidad de Murcia, España. 663-677 p.

ProDUS, (2015). Plan de Desarrollo Turístico Sostenible del distrito de Curubandé, cantón de Liberia. 325 p.

Universidad Nacional de Costa Rica UNA. (2012). Propuesta de Plan Regulador para el distrito de Curubandé. Diagnóstico Sociocultural, Económico, Turismo y Migración. 292 p.

Zeledón Angulo, María Gabriela (2011). Iconografía de los petrograbados ubicados en la sección del Pie de Monte Suroeste del macizo Volcánico Rincón de la Vieja. Trabajo final de Graduación para Licenciatura antropología con énfasis en Arqueología, Montes de Oca, Universidad de Costa Rica. 